\title{
Digital surveying and 3D modelling structural shape pipelines for instability monitoring in historical buildings: a strategy of versatile mesh models for ruined and endangered heritage
}

\author{
Sandro Parrinello' ${ }^{1}$ Raffaella De Marco ${ }^{1}$ \\ ${ }^{1}$ DICAR - Department of Civil Engineering and Architecture, University of Pavia, via Ferrata 3, 27100 Pavia, Italy
}

\begin{abstract}
Cultural heritage and the attendant variety of built heritage demands a scientific approach from European committees: one related to the difficulties in its protection and management. This is primarily due to the lack of emergency protocols related to the structural knowledge and documentation pertaining to architecture and its ruins, specifically in terms of the goals of protection and intervention for endangered heritage affected by mechanical instabilities. Here, we focus on a rapid and reliable structural documentation pipeline for application to historical built heritage, and we introduce a case study of the Church of the Annunciation in Pokcha, Russia, while we also review the incorporation of integrated 3D survey products into reality-based models. This practice increases the possibility of systematising data through methodological phases and controlling the quality of numerical components into 3D polygonal meshes, with millimetric levels of detail and triangulation through the integration of terrestrial laser scanner and unmanned aerial vehicle survey data. These models are aimed at emphasising morphological qualities related to structural behaviour, thus highlighting areas of deformation and instability of the architectural system for analysis via computational platforms in view of obtaining information related to tensional behaviour and emergency risks.
\end{abstract}

Section: RESEARCH PAPER

Keywords: Endangered Heritage; reality-based modelling; digital survey; structural modelling; Cultural Heritage Routes

Citation: Sandro Parrinello, Raffaella De Marco, Digital surveying and 3D modelling structural shape pipelines for instability monitoring in historical buildings: a strategy of versatile mesh models for ruined and endangered heritage, Acta IMEKO, vol. 10, no. 1, article 12, March 2021, identifier: IMEKO-ACTA$10(2021)-01-12$

Editor: Eulalia Balestrieri, University of Sannio, Italy

Received June 1, 2020; In final form November 23, 2020; Published March 2021

Copyright: This is an open-access article distributed under the terms of the Creative Commons Attribution 3.0 License, which permits unrestricted use, distribution, and reproduction in any medium, provided the original author and source are credited.

Funding: This project has received funding from the European Union's Horizon 2020 research and innovation programme under the Marie Skłodowska-Curie grant agreement No 821870 .

Corresponding author: Raffaella De Marco, e-mail: raffaella.demarco@unipv.it

\section{INTRODUCTION: THE DOCUMENTATION OF ENDANGERED HERITAGE}

Both the analysis of cultural heritage and the development of communitarian guidelines for its protection [1] are determining a growing scientific approach to European and worldwide heritage sites [2]. The variety of built heritage (historical buildings, monuments, historical centres, sites, and territorial landscapes) requires a wider field of knowledge and intervention in terms of both physical structures and cultural policy. This leads to the attendant difficulties in protection and preservation due to a fragmented reality of separated protocols and documentation, which results in difficulties in the sharing of information and data integration, thus hindering the entire approach to an intervention programme. This is especially the case for the so-called 'endangered heritage' sites (World Heritage Convention, List of World Heritage in Danger 1972), the class of built heritage particularly affected by proven or potential threats that pose a high level of risk for its preservation [3].

In fact, the revision of the sites officially listed as endangered heritage has been achieved in terms of the conditions of relevance and correspondence with the specified risk criteria and indicators (territorial, political, or social dangers). However, the statistics in terms of monitoring parameters and protocols remain non-uniform, highlighting a lack of scientific and technical documentation and a lack of connection between intervention activities and 'reality-based' updated analysis. The framework highlights the coexistence of a dual type of emergency issue related to built heritage, with the classification 

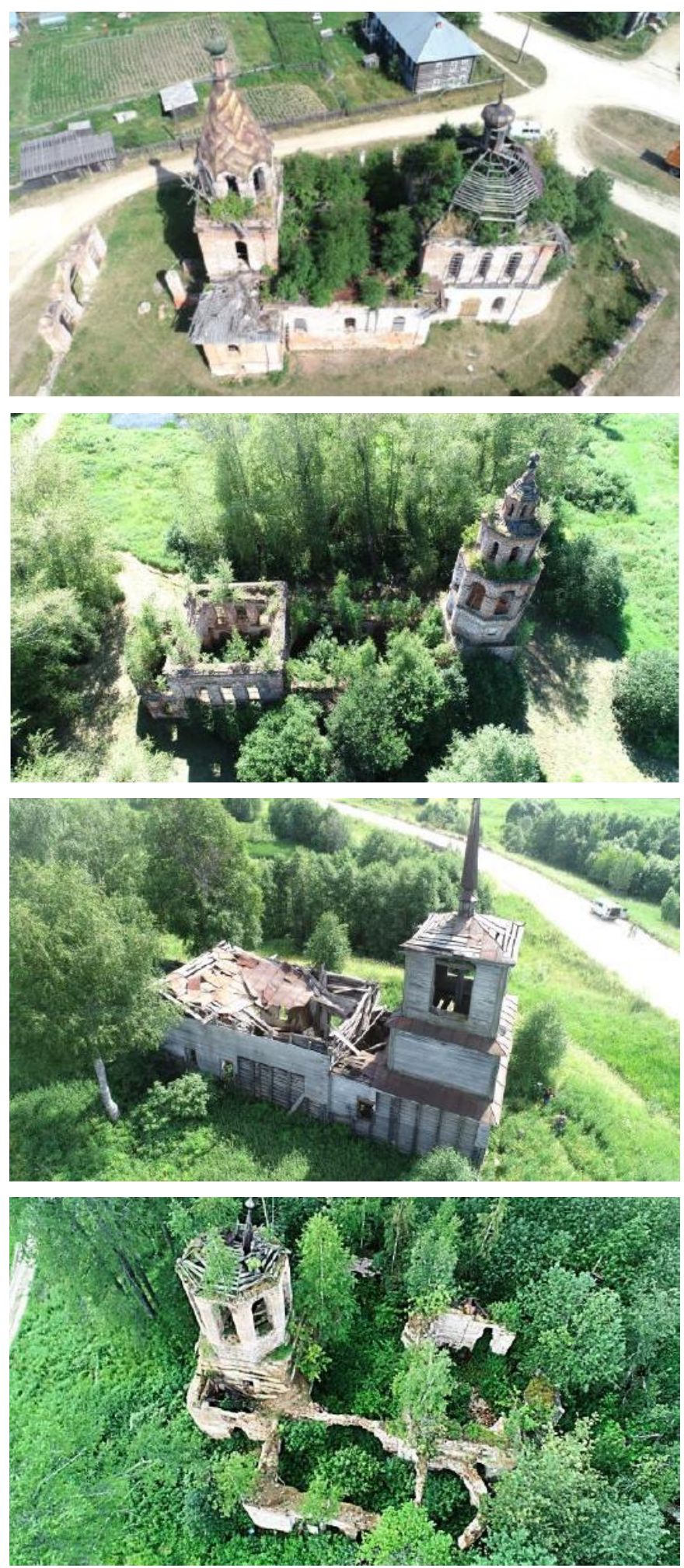

Figure 1. Examples of sites along the Upper Kama route characterised by ongoing risks of abandonment and decay, proving the basis for a classification as endangered heritage. From the top, Church of the Exhaltation of the Holy Cross in Bondjug, Rubezhskaya Church in Usolye, Church of St. Nicholas in Uzhginskay, Church of Paraskeva Friday in Saltanovo.

of the site as 'endangered' for the stability and integrity of its physical

structure on the one hand, and the growing demand for specific parameters and protocols for the analysis and quantification of the emergency value of the building, thus determining the effective intervention.
Thus, there is a growing demand for the identification of such sites [4], both in geographical and typological terms, expanding the dissemination and application of appropriate monitoring and knowledge practices prior to intervention [4], with the aim of triggering a process of safeguarding policies [6].

The topic of implementing safeguarding methodologies for existing heritage is receiving a great deal of attention among researchers, with new forms of digital products emerging in terms of a survey-based phase of digital documentation related to cultural heritage, which is crucial for an accurate and complete understanding of the characteristics and parameters of the units and contexts of built heritage. This is being combined with computer-based cognitive and interactive models, with the elaboration of 3D digital products for investigations and simulations related to shapes and structures.

These new representation systems are resulting in new expectations related to digital communication, changing the objectives and constantly renewing the demand in analytical terms for cognitive requirements, in response to the requirements linked to the computational nature of interaction within the models themselves, which are now capable of providing both quantitative and qualitative answers. The difficulties in the development of the reliable diagnosis of historical structures can be realigned with the need for new methods of analysis that can exploit the computational opportunities through specific methodologies and cognitive practices. These practices relate to the visual and graphic aspects of the documentation pertaining to architecture [7], reliably representing the present state of stability and linking it to the constructive and safeguarding rules.

\section{HISTORICAL STRUCTURES AND INSTABILITIES IN THE DIGITAL DOCUMENTATION PROCEDURES}

Historical buildings are traditionally founded on and remain linked to their original structural system, from the construction phase to later procedures. The demolition of bearing systems and elevations, the reconfiguration of horizontal levels and vaults, and the construction of expansion blocks thus enrich the mechanical experience of the monument (Figure 1).

The consideration of structural diagnosis applied to historical built heritage in terms of the knowledge of the stress behaviours and the prevision of damage mechanisms plays a central role in the development of the documentation protocols for safeguarding. A review of the risks, the on-going kinematics, and the priorities of stability related to endangered buildings [8] places the focus on the aspect of robustness, that is, the capacity of the building and its elements to withstand a certain level of stress imposed by a combination of degradation and the changes in both the materials and the environments [9]. Thus, considering the deep impact that stress-altering phenomena have on the geometrical and material aspects of buildings (e.g. deformations, drifts, cracks) is part of a comprehensive diagnosis that can be achieved through an investigation into the structural shape [10]. As such, it is possible to focus the evaluation of the stress instabilities on the target of a 'static morphology' that reflects the mechanical and stress traces left by the adaptation schemes of the system during its physical history. It also highlights the stability principles related to the class and type of resistant geometries (Figure 2).

Nowadays, the analysis of the structural aspects of historical buildings involves simplified typological schemes transformed into numerical structures, selected to be mechanically 
controllable in defined levels of physical regularisation and abstraction [11]. However, the confidence in the standardisation of structural computing has led to underestimating the direct 'experience' of the building, visible in the singular imperfection of its shape.

In fact, structural documentation must be both 'comparative', with reference to the archetypes, and 'specific', highlighting the accurate variation in formal detail. The testing of specific intervention procedures for existing components aimed at assessing their instability performance is highlighting the need for more precise models for the creation of reliable simulations [12]. At the same time, the area of digital documentation has developed to include morphometric 3D databases with more dense information, which allows for measuring and analysing space in terms that is closer to the continuous form [13]. The relationships between the shape and mechanics of structural systems can determine new descriptive products through realitybased numeric surfaces in terms of high-poly mesh models, which can be morphologically assessed to extract specific quantitative parameters that can guide the mechanical interpretation. Thus, the reality-based mesh model, the numerical component of which reliably determines and characterises the geometric aspects, can become an adaptable tool for the management of the computational and analytical processes related to robustness.

These considerations are encouraging strategies of data integration for the comprehensive adoption of structural realitybased models related to architectural heritage. On a scientific level, the experimentation of reality-based models for structural diagnosis will result in a multidisciplinary and implementable methodology, one capable of guaranteeing a standardised product, that is, the polygonal mesh model, involving different levels of detail and integration for the management of the existing built heritage. This type of model is aimed at determining both emergency and long term interventions, in the calibration of their procedural computing in both a scientific and practicaloperational direction through the capacity for the shapes to be transformed into morphological and computational platforms of analysis, such as finite element analysis (FEA) platforms. Here, the methodological process must be as fast, as extendable, and as replicable as possible to facilitate interchange and to allow for a complete knowledge and management capacity through 3D models related to buildings that are in a state of emergency.

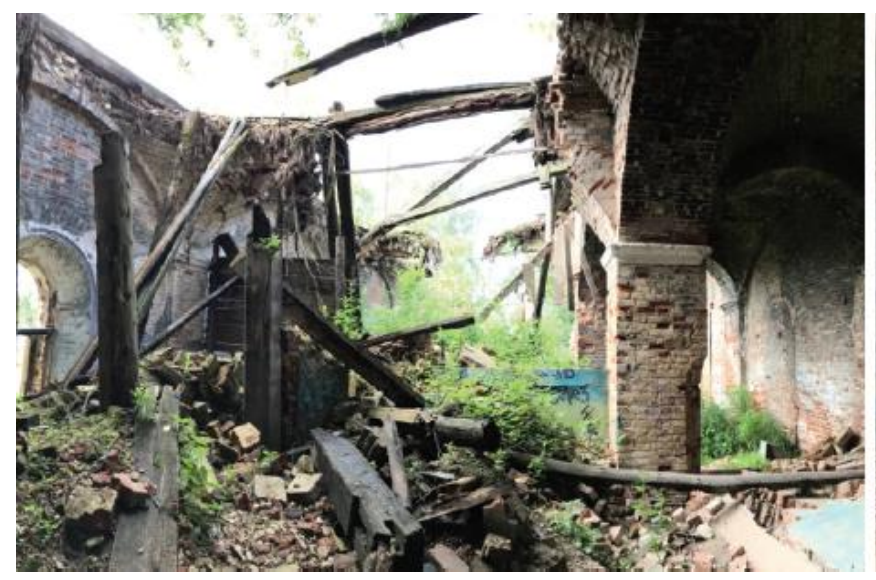

\section{AN EXPERIMENTAL CASE STUDY: THE CHURCH OF THE ANNUNCIATION IN POKCHA (RUSSIA)}

\subsection{The site and its historical/structural characterisation}

The constructive features of built structures relate to the technological and material traditions pertaining to specific continents, where the stylistic influences and source availability of certain elements have determined the specific characteristics of the architectural shape. This is especially the case with the built sites and monuments along the European Cultural Heritage Routes, the architectural value of which is strongly permeated by specific structural typologies. These architectural structures have thus acquired their own identity, characterisation, and specificity over time, evolving specific spatial distributions and constructive apparatuses in their historical development and functional change. Resilient structures combine a wide framework of morphological and static relationships that are reflected in their own state of conservation and which influence the robustnessrelated approach to their restoration and preservation for security and stability.

The case of Upper Kama, Russia, already the subject of international research related to the digital documentation of the complexes [14], presents an emblematic example of the Cultural Heritage Route and the coexistence of historical structures and various phenomena of instability caused by the functional experience of the building, reflecting a complex framework of diagnosis for the safety and integrity of the various built sites. In these terms, the Upper Kama sites form a rich morphological abacus of technological modules and elements of structure, along with the related pathologies of degradation and conservation [15] [16].

The cultural tradition of the Upper Kama settlements, more stabilised by commercial requirements than most villages of the Russian countryside, has determined a global phenomenon of transformation regarding the built structural systems, from the typical wooden architecture to the constructive solutions of brick and stone masonry. Therefore, these architectural structures are characterised by a morphological configuration involving multiple expansion blocks. During the Soviet period, the demand for the conversion of infrastructures for energy or food production resulted in the decline of these religious buildings. The consequent interventions and the alteration of the architectural structures, forcibly adapted to the new functions, led to a prevalent abandonment characterised by eventual ruin and collapse.

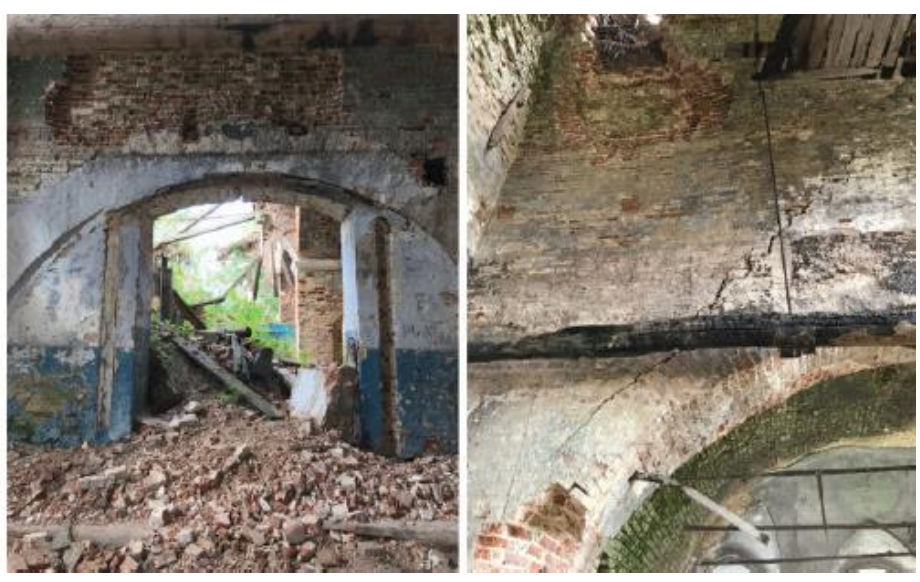

Figure 2. The state of neglect and the lack of conservation present critical situations of widespread instability among the masonry systems, clearly exposing the structure to the damage affecting its elements. Robustness instabilities in the structural complex of the Church of the Transfiguration, Pyskor, defining the increasing frameworks of damage that will destroy these buildings without specific lines of intervention. 
The case of Blagoveshchenskava Church, or the Church of the Annunciation of the Blessed Virgin, in the village of Pokcha, represents a key monumental site of the Cherdyn district, which synthesises various different historical-functional evolutions in the stratification of its structures and walls, which today lie in ruin [17], Figure 3.

The original wooden complex was replaced in 1785 with a new structure composed of stone and brick masonry, subdivided into multiple structural blocks, which include the main body, with a quadrilateral planimetry, constituting a nucleus for the refectory, the southern and northern chapels, the bell tower, and the entrance narthex. In 1910, a reconstruction intervention resulted in various structural modifications, especially in terms of the bell tower, which was entirely replaced, and the eastern section of the central vault and the altar, reconstructed with the insertion of a five-headed chapter. Meanwhile, the interiors formed in plastered stone, with paintings and ornaments dating from 1870 , remain largely preserved. The face of the building, which features an additional red brick facing, contributes to the strengthening of the external envelope and allows for the possibility of inserting additional devices of tension resistance into the stratified walls.

The history of the site involves attempted restoration works dating from 1920 to the complete abandonment in 1940 and to the re-conversion into a power central. The energy issues related to the new function resulted in the partial collapse of the main pavilion vault and the bell tower roof in the 1990s, following repeated lightning strikes attracted by the electrical system. Following this extensive damage, the church was excluded from the list of architectural monuments of interest, precluding any new interventions or restoration works, and leaving the site to suffer virtual collapse.

\subsection{The current state of the structural ruins}

In 2018, the architectural complex was in a clear state of neglected conservation. The wooden roofs had been almost entirely destroyed, and the main masonry structure was damaged in various areas, particularly in the main vaulted system.

Meanwhile, the vaulted structure of the spans, the loading on the pillars based on a counter-balance scheme involving vaultvault and vault-support relationships, has been distorted by the collapse of the secondary vaults. In terms of the large central pavilion vault, only around half of its total span has been preserved, with the head brick structure left visible along the detachment edge. Both the brick vaults and the wooden roof have collapsed into the central environment of the nave, with the debris submerging part of the loading pilasters of the vaults, which cannot be inspected in their ground basement and have been covered by soil and vegetation, creating a natural hill that both reduces the access and is the cause of the degradation of the preserved supports. The connection with the bell tower, once provided by the central nave through the gallery and the refectory, has been demolished and thus prevents the direct documentation of the state of conservation of the elevated structures.

Following the collapse of the roof, the complex was deprived of the main source of protection from atmospheric agents, particularly pertinent during the winter months, and has therefore suffered a rapid degradation of the remaining portions, affected year on year by localised collapses. The site is also totally lacking in control services to prevent access to both people and animals, who often occupy it and damage the spaces and structures. Here, the narthex environments have been
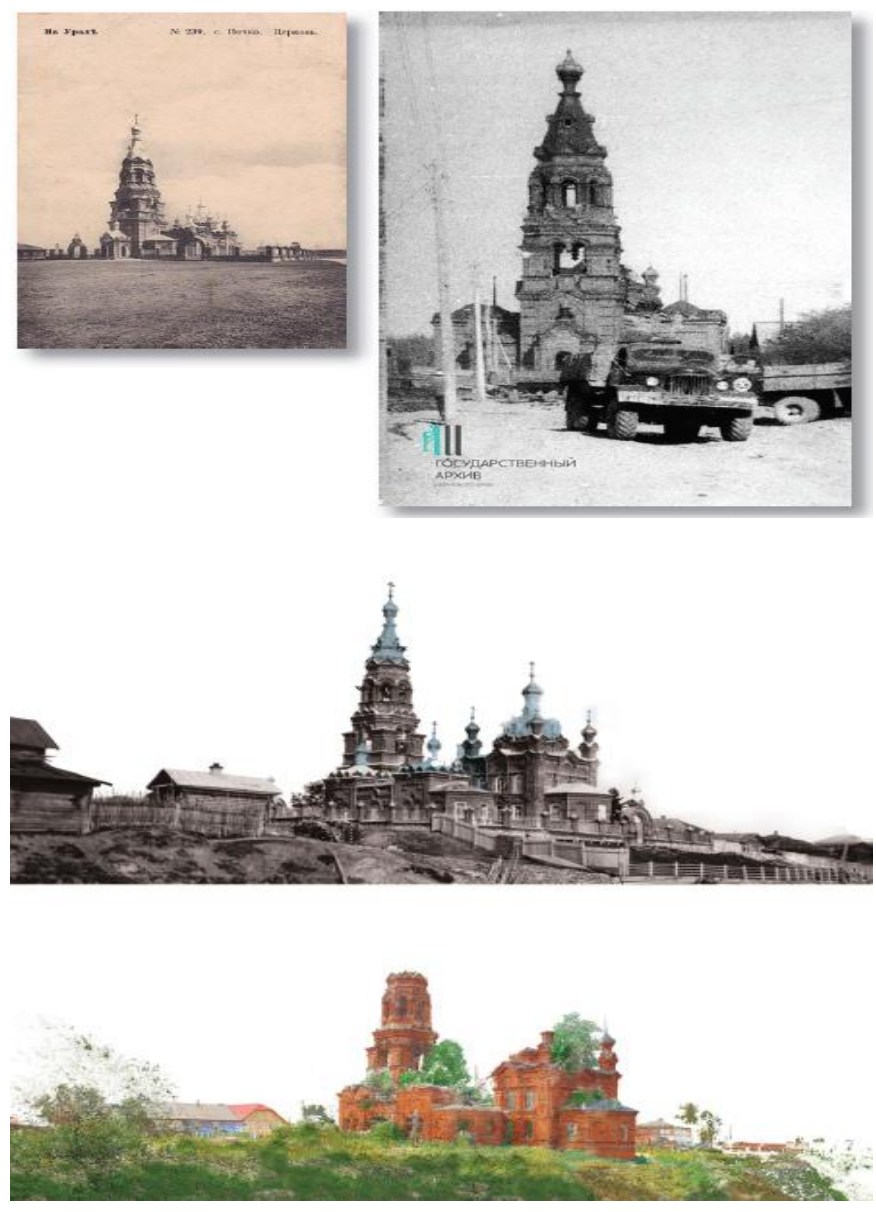

Figure 3. The complex of Blagoveshchenskaya Church in Pokcha. The original architectural structure of the church before the revolution in 1956 (above) and the ruins as of 2018 (below).

particularly affected by the frequent presence of herds in transhumance, often housed by the shepherds inside the church during the summer season (Figure 4).

\subsection{Objectives and targets of the documentation process}

The objective of the documentation of the Blagoveshchenskaya Church, in addition to the wider mapping and reconnaissance approach for the sites in the Upper Kama area, is to encourage a rapid but reliable diagnosis of its structural crisis. This is aimed at directing the organisation of possible intervention operations, and to safely preserve and promote the historical ruins. While the local administration is gradually recognising the historical and cultural value of these sites along the Kama, the ruined conditions present a clear disadvantage for the commitment, both technical and economic, to implementing conservation procedures. In this sense, rapid survey and quantification methods for recognising and evaluating the preserved structural systems and the corresponding risk constitute an excellent preliminary tool for accurately determining the qualitative and quantitative planning of interventions that could halt the exponential cycle of damage.

The documentation of the current state of conservation of the structure of the Blagoveshchenskaya Church has thus highlighted the need for structural diagnosis using 3D digital survey methods. This involves applying specific integrated approaches for the acquisition and modelling of the structural shape, one that ensures a descriptive quality of its resistant architectural structure through the data collected through a 

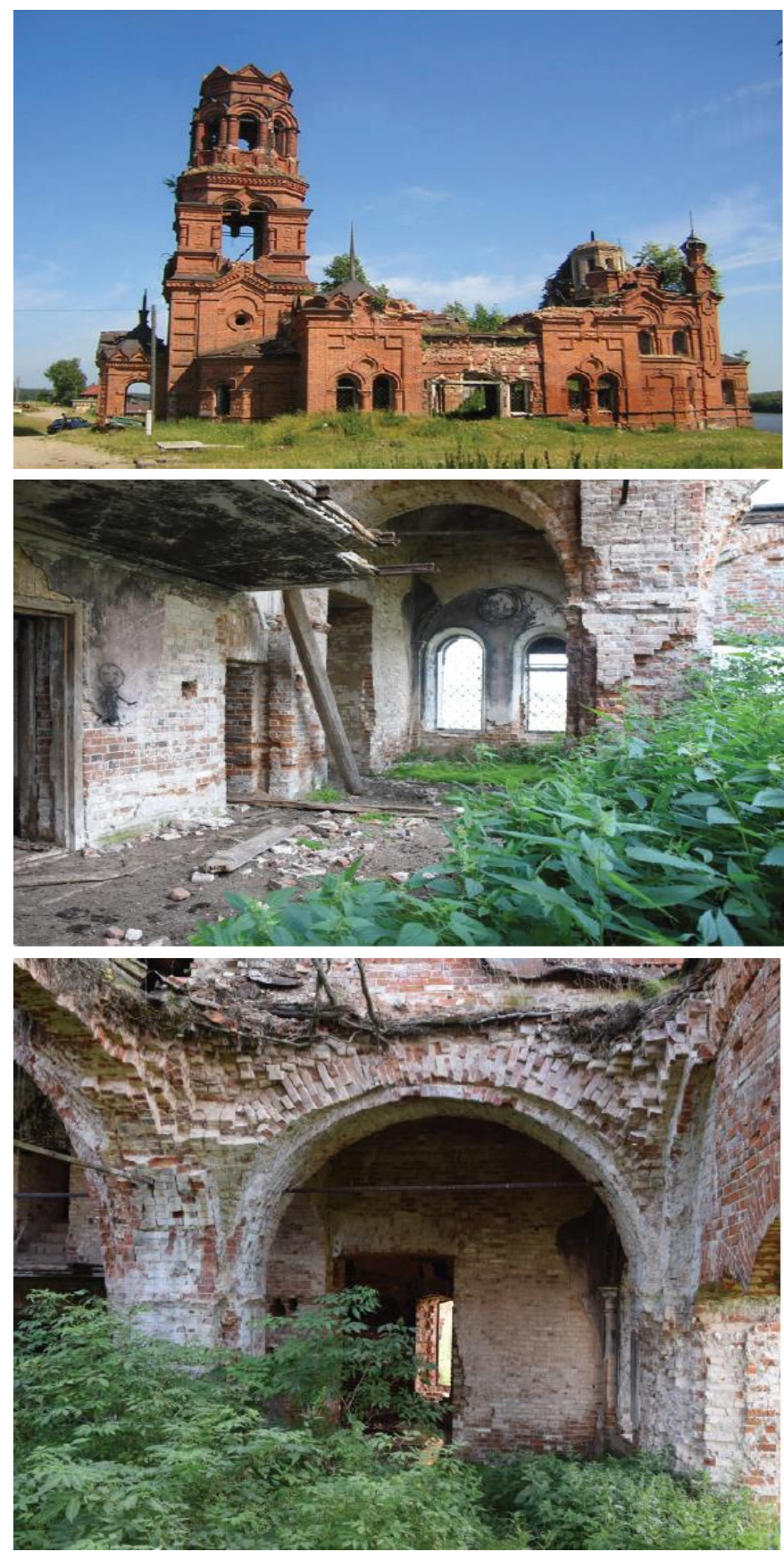

practical and fast on-site survey. The required knowledge is largely related to the shape alteration of the structural ruins, ensuring that it can be comprehensively and reliably documented and allowing for analysing the degree of safety and determining the recovery intervention in relation to the overall mechanical scheme.

\section{STRATEGIES OF ANALYSIS FOR THE DIGITISATION OF THE RUINED SHAPE}

The documentation of the state of conservation of Blagoveshchenskaya Church as of 2018 involved experimenting with integrated approaches in digital surveying to focus on the static instabilities of the ruins for recovery purposes [18]. Here, the morphological analysis of masonry structures was organised during the acquisition processes, semantising the spatial constructive ruins and linking them to the global volumetric macrosystem. Furthermore, the internal inspection of the
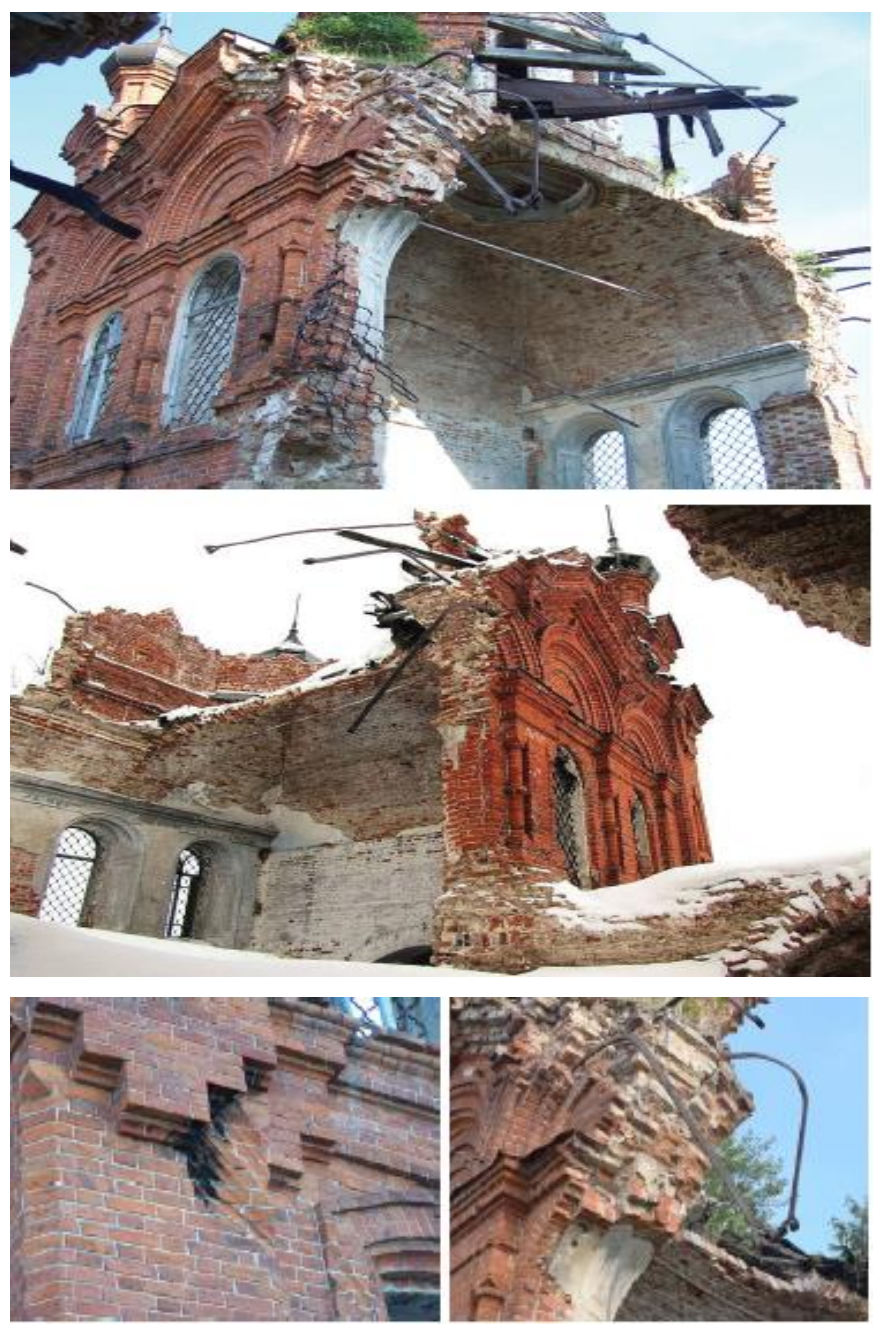

Figure 4. Photographic gallery of the Blagoveshchenskaya Church in Pokcha. On the left, the state of conservation in 2018, the overall structural system and the specific static units that characterise the statics of the entire complex. On the right, evolution of the damage to the main vault from 2006 to 2019, which increased rapidly due to the lack of intervention. Details of the constructive system that influences the robustness evaluation of the complex. Indication of interventions for the functional conversion into a power central, stratigraphic technology of the masonry walls and distribution of a steal structure inside the masonry.

masonry sections in terms of the fractured or collapsed portions has allowed for the integration of information related to the structural envelope, reliably supporting the digitisation procedure [19].

The ruined shape, the central element of the analysis process, has thus become the basis of direct knowledge, supported by the repertoire of historical/constructive analysis data, with the dual purpose of completing the technical stratigraphic knowledge framework and revising it using integrated research in relation to the behavioural diagnosis of the structural components. The ruined complex of Blagoveshchenskaya Church, for reasons attributable to the collapse of the structures and the lack of site regulation, has been deprived of the original spatial design of the wall system. The absence of the main masonry portions hinders the analysis of the current structure in terms of creating defined schemes of interpretation in relation to the architectural instabilities, and this indicates that the specific evaluation of the 
reality-based digital shape is a crucial step for a reliable diagnosis of the static robustness [20].

The ruined complex has been subjected to an integrated, extensive, range- and image-based documentation campaign. The attendant approach involved calibrating methodologies of acquisition and representation in terms of the morphological properties of the structural elements, especially in terms of the correspondence and integration of spatial information. The dimensions of the structure (specifically the elevation of the damaged portions of the main vault at over $15 \mathrm{~m}$ in height) have oriented the morphometric digitisation strategy toward the use of multi-instrumental close-range measuring systems, aimed at guaranteeing a comprehensive coverage and a high formal detailed resolution comparable to the complexly shaped geometry of the masonry surfaces and the typological damage of the masonries (widely covering the order of dimensions from 5 to $10 \mathrm{~cm})$.

At the same time, the colorimetric information has proven to be a key aspect in the diagnostics of the structures, evaluating the pathologies of mechanical influence on the masonry and plastered portions, described through tonal variations in the surface colours.

The on-site campaign was organised on a double acquisition level, at the ground level using a terrestrial laser scanner (TLS) and at an aerial level using drones for photogrammetry, with the aim of obtaining comprehensive morphological data on the elevations of the structure (over $15 \mathrm{~m}$ ) in a single $360^{\circ}$ point cloud database [21].

\subsection{Terrestrial laser scanning acquisition campaign and products}

In the acquisition using a TLS FARO Focus S150, the integrated photographic camera was also enabled, ensuring a red, green, blue (RGB) information quality for each point ( $x, y, z)$ that is suitable for surface mapping. Each scan was performed in medium or high mode according to the distance of the shooting range from the object and provided an additional acquisition time of 4.00 minutes to allow for the shooting of 16 frames for each camera location (coverage of $360^{\circ} \times 320^{\circ}$ for the scan angle). Prior to each photographic shot, the instrument was set with an average balance of colour and lighting while considering only the horizontal level for the balance of light conditions in the outdoor scans, or the entire panoramic space for the interior scans. These measures significantly affected the acquisition campaign in terms of duplicating the scanning times but were still more advantageous for the documentation purposes than a parallel photographic campaign from the ground, which requires further actions to integrate the photographic material into the spatial database. A total of 73 TLS scans were realised to collect all external surfaces and to spatially connect the complex distribution of the internal environments. The scans were performed at an approximate $2-\mathrm{mm}$ laser spot spacing up to a height of $5 \mathrm{~m}$, and close to $5 \mathrm{~mm}$ in the upper surfaces. The quality of TLS acquisition for the bell tower and the central dome was ensured by the presence of the inner natural hill over the ruins of the roof, which allowed for a higher instrument positioning above ground level. The TLS metric survey conducted from the ground guaranteed a coverage of $80 \%$ of the morphological data related to the structure (Figure 5).

The processing of the seven source scans on the vaulted structure provided an alignment procedure to obtain the unified TLS point cloud. Rotation and translation matrixes were then applied in semi-automatic mode via the cloud software (SCENE), using a cloud-to-cloud alignment set with values of
Table 1. Alignment values in cloud-to-cloud reference for TLS data.

\begin{tabular}{lcccc}
\hline Scan & $\begin{array}{c}\text { Morpho } \\
\text { feature } \\
\text { iterations }\end{array}$ & $\begin{array}{c}\text { Point error } \\
\text { iterations } \\
\text { in } \mathbf{~ m m}\end{array}$ & $\begin{array}{c}\text { Average point } \\
\text { error } \\
\text { in } \mathbf{~ m m}\end{array}$ & $\begin{array}{c}\text { Min } \\
\text { overlapping } \\
\text { in \% }\end{array}$ \\
\hline Scan 39 & 4 & 2.5 & 2.3 & 35.5 \\
Scan 40 & 12 & 2.6 & 1.8 & 25.8 \\
Scan 41 & 7 & 1.9 & 1.6 & 29.5 \\
Scan 42 & 6 & 1.5 & 1.2 & 29.5 \\
Scan 43 & 5 & 1.2 & 1.0 & 49.4 \\
Scan 46 & 6 & 1.3 & 1.0 & 33.8 \\
Scan 48 & 4 & 2.2 & 2.0 & 26.3 \\
Cluster report & 44 & 1.8 & 1.5 & 32.8 \\
\hline
\end{tabular}

$0.05 \mathrm{~m}$ in subsampling and $\mathrm{n}^{\circ} 150$ iterations for the alignment algorithm. The resulting values of alignment characterising the final integrated database are presented in Table 1.

\subsection{Unmanned aerial vehicle acquisition campaign}

At the same time, the aerial photogrammetric acquisition was developed using an unmanned aerial vehicle (UAV, DJI Phantom 4 Pro), with the flight plan focused on the points of interest of the architectural complex. In fact, the flight plan mission was set from the top centre of the complex at a level of $50 \mathrm{~m}$ from the ground in the mode 'point of interest'. Here, a photogrammetric campaign was conducted using the aerial camera with a conical acquisition surrounding the monumental complex, descending to a height of $10 \mathrm{~m}$ above ground and developing 329 shots in 10 min of flight. The guaranteed camera angle and the adjustable inclination of the gimbal allowed for total coverage of the top surfaces, with a quality of photographic data suitable for the clear photogrammetric reconstruction of the edges of the components (21 MPixel). In addition, specific flight plans for the tower blocks and the central vault were planned with greater photographic detail, largely due to the close distance of shooting. The GPS coordinates defined by the UAV for each shot better addressed the alignment algorithms, set to 'high' precision, simplifying the alignment of the photogrammetric structure from motion (SfM) reconstruction. In terms of the overall SfM point cloud, there was an error of 0.004 pixels due to the camera alignment (Figure 6).

\subsection{Post-processing data and products}

The GPS information in the source data obtained via the acquisition campaign provided a preliminary overlapping between the TLS- and UAV-acquired point clouds, both in terms of vertical and horizontal built surfaces, which was used to optimise the referencing in an integrated sparse database of morphometric characters.

To optimise the GPS data, the alignment procedure for the TLS and UAV data was set with reference to the TLS point cloud, defining six markers from key morphological features that were recorded on the UAV point cloud. The resulting values of alignment characterising the final integrated database are presented in Table 2. The higher errors of reference corresponded to the markers with less camera projection in terms of photogrammetric alignment.

The final database featured 51.889 .557 and 49.853 .646 points from the TLS survey on the internal surfaces of the masonry structure, and 2.035.911 points from the UAV survey on the external surface and the ruins of the vaulted structure. The average density in the overall integrated point cloud was 1 point $/ 3 \mathrm{~mm}$. 

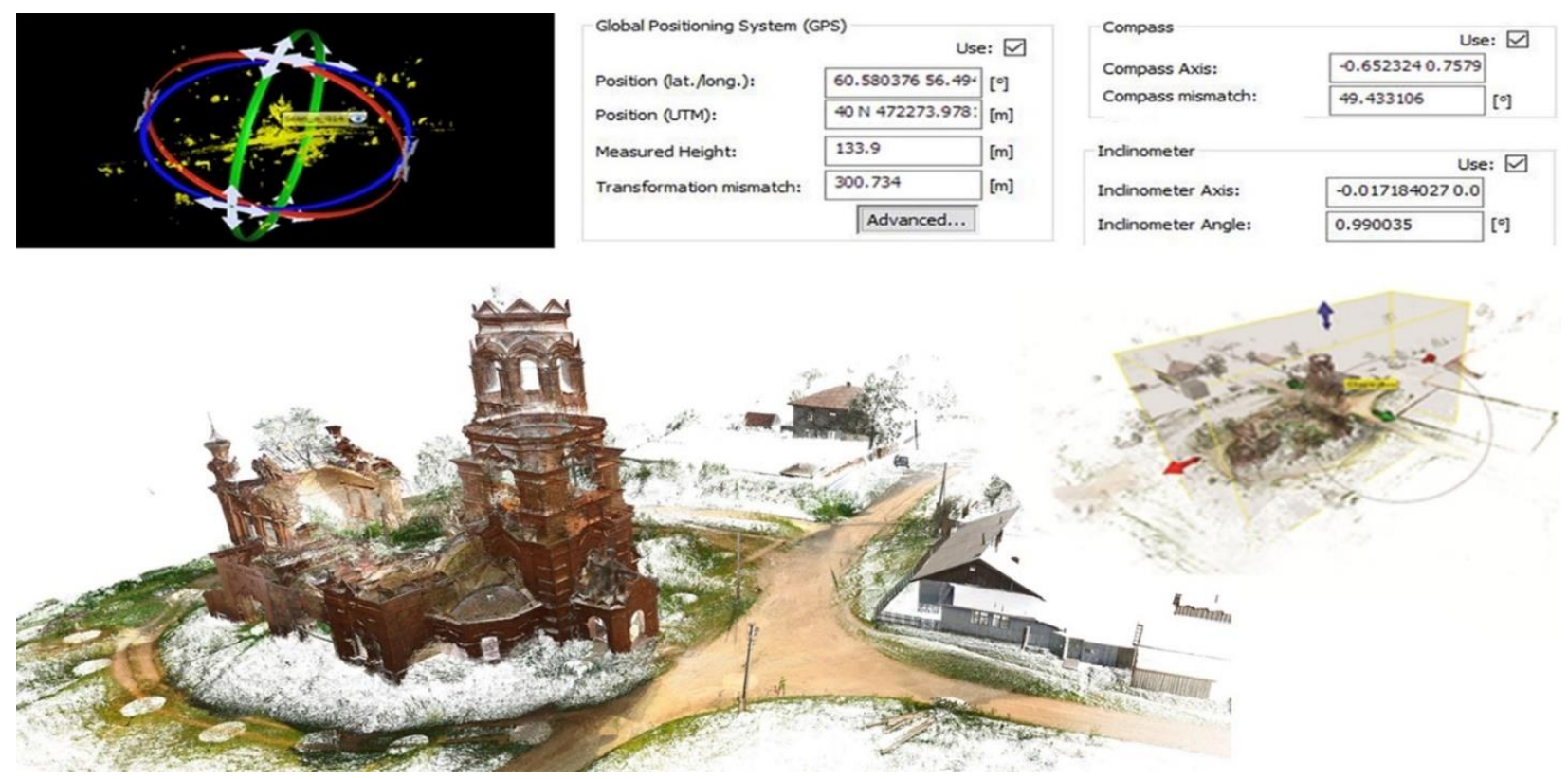

Figure 5. Morphometric database from on-site TLS acquisition campaign: the single scans, completed via GPS, compass, and inclinometer information, were referenced and registered to control the overall alignment to verify the final point cloud.

Table 2. Alignment values in TLS and UAV data morphometric reference.

\begin{tabular}{lccc}
\hline Marker & $\begin{array}{c}\text { Morpho feature } \\
\text { correspondence }\end{array}$ & Projections & Error in $\mathbf{m}$ \\
\hline Point 1 & Vault springer 1 & 89 & 0.0383 \\
Point 2 & Vault springer 2 & 130 & 0.0472 \\
Point 3 & Vault springer 3 & 114 & 0.0483 \\
Point 4 & Vault springer 4 & 85 & 0.0439 \\
Point 5 & $\begin{array}{c}\text { Vault key 1 } \\
\text { (damaged border) } \\
\text { Voint 6 }\end{array}$ & 87 & 0.0314 \\
Control key 2 & 77 & 0.0169 \\
\hline
\end{tabular}

From the joint processing of the collected archives, useful reference drawings, sections, and plans were produced to obtain comprehensive knowledge of the architectural complex and to serve as guidance for the intensive mapping of the pathologies of degradation and instability documented in the photographic repertoire. However, the $2 \mathrm{D}$ representation barely described the quality of distribution and the volumetric development of the main structural elements, rendered even more complex by the ruined condition of the site and, as such, by the loss of many of the main shapes conventionally identifiable for the classification of the elements (intrados of the vaults, opening profiles and local collapses, integrity of the elevation structures).

In addition, the attempt at processing for elevation maps, as a standard analysis of deformation mechanisms using the point

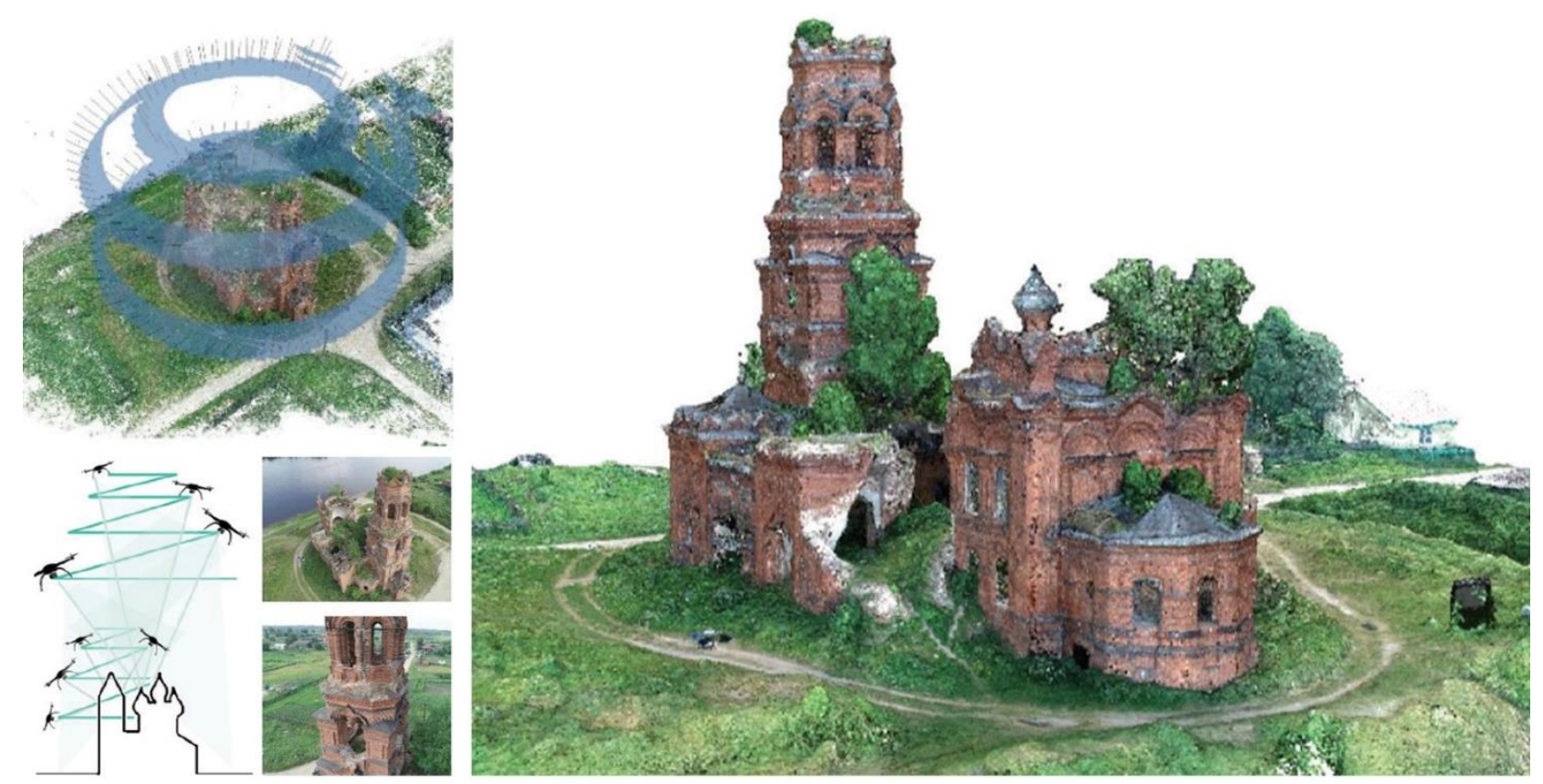

Figure 6. Morphometric database from the on-site photogrammetric UAV acquisition campaign: the photos were geo-referenced and aligned to define the main point cloud on the architectural complex and the surrounding landscape. 
cloud displacement from a reference plane [22], did not satisfy the knowledge requirements in terms of the deformation of the structural surfaces. The high surface processing of the structural envelope, due internally to the collapse conditions and externally to the decorative brick structure, resulted in difficult-to-read colour maps, where the excessive fragmentation of the level indicators failed to provide an overall interpretative picture of the deformations.

\section{OPPORTUNITIES FOR 3D INTEGRATED MODELLING FOR THE DIAGNOSIS OF THE STRUCTURAL SHAPE}

The aim of the structural representation was focused on the development of a continuous mesh modelling protocol, enriched with reality-based qualities, achieved as part of the objective of digitally surveying detailed and imperfect shapes as confirmed by the instability and mechanical features. These shapes, controlled and certified within the envelope of the structural object, necessarily introduce a need to manage the polygonal geometric mesh that defines them, the processes of which are, however, improved by the representation of a morphological detail that is itself a source of diagnostic reflections on the mechanisms of analytical systems [22] [24], Figure 7.

In this sense, the obtained 3D models not only pursue the necessary analysis of the deviation between static surfaces but also consolidate a certified practice that defines them both as outputs, directly applicable for the quality of structural instabilities, and as input data for further implementation, specifically on computational platforms.

As such, a digital data processing was established that will exponentially led to the exploitation of the potential of the $3 \mathrm{D}$ discrete database, namely, the point cloud, and the continuous $3 \mathrm{D}$ object defined by vertex and edges for quantifiable evaluations, namely, the model. The application opportunities, from FEM platforms to information systems that enhance the virtual fruition, can thus provide the grounding for involving the models using common languages, thus adapting them appropriately [25] [26], Figure 8 and Figure 9.

The developed research on reality-based mesh models has allowed for accurately delineating the causes and effects produced by the structural survey in the digitisation and modelling processes related to morphometric data, validating the compatibility and correspondence of the produced models via a multi-instrumental comparison of the measurement practices normally applied in the structural field. The integration of the products of digital survey protocols applied on the site [26], from both terrestrial and aerial image-acquisition tools, was complementarily completed through the differentiated visual stations, ensuring the capacity to obtain information related to both the exterior and the interior parameters, as well as to monitor the data related to the roof components and elevation units. Focusing on the central pavilion vault, which was largely destroyed during the electrical accident, the documentation, finalised to the reinstatement of a complete structural shape, involved the detection of the vault both from the intrados level, also visible in terms of its constructive thickness, and the extrados level, dominated by the ruins of the octagonal masonry tholobate at the base of the wooden roof, Figure 10.

\subsection{Modelling pipeline}

The pipeline for 3D reality-based modelling was assessed in relation to the research objectives regarding the diagnosis of the stability of the Blagoveshchenskaya Church in Pokcha. The
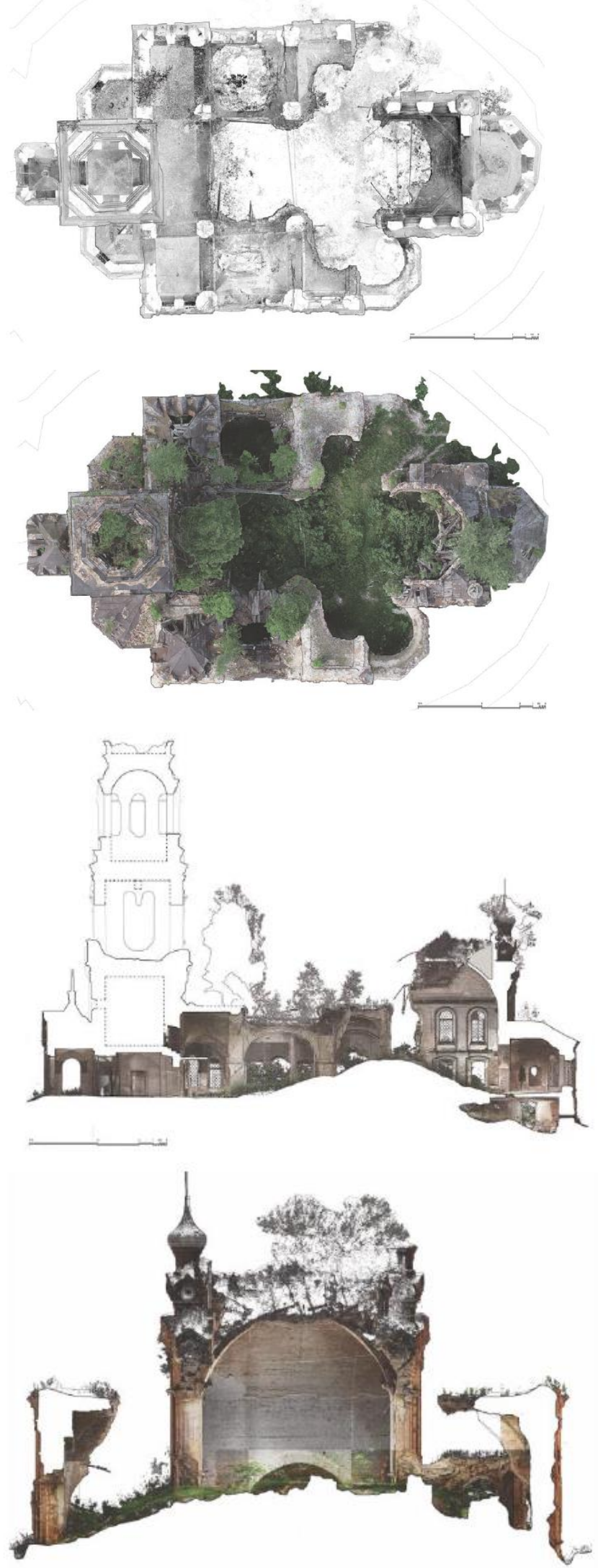

Figure 7. Drawings and elaborations for the 2D description of the main structural system of the church. The presence of noise data for elements such as the vegetation and collapsed ruins is highlighted. 

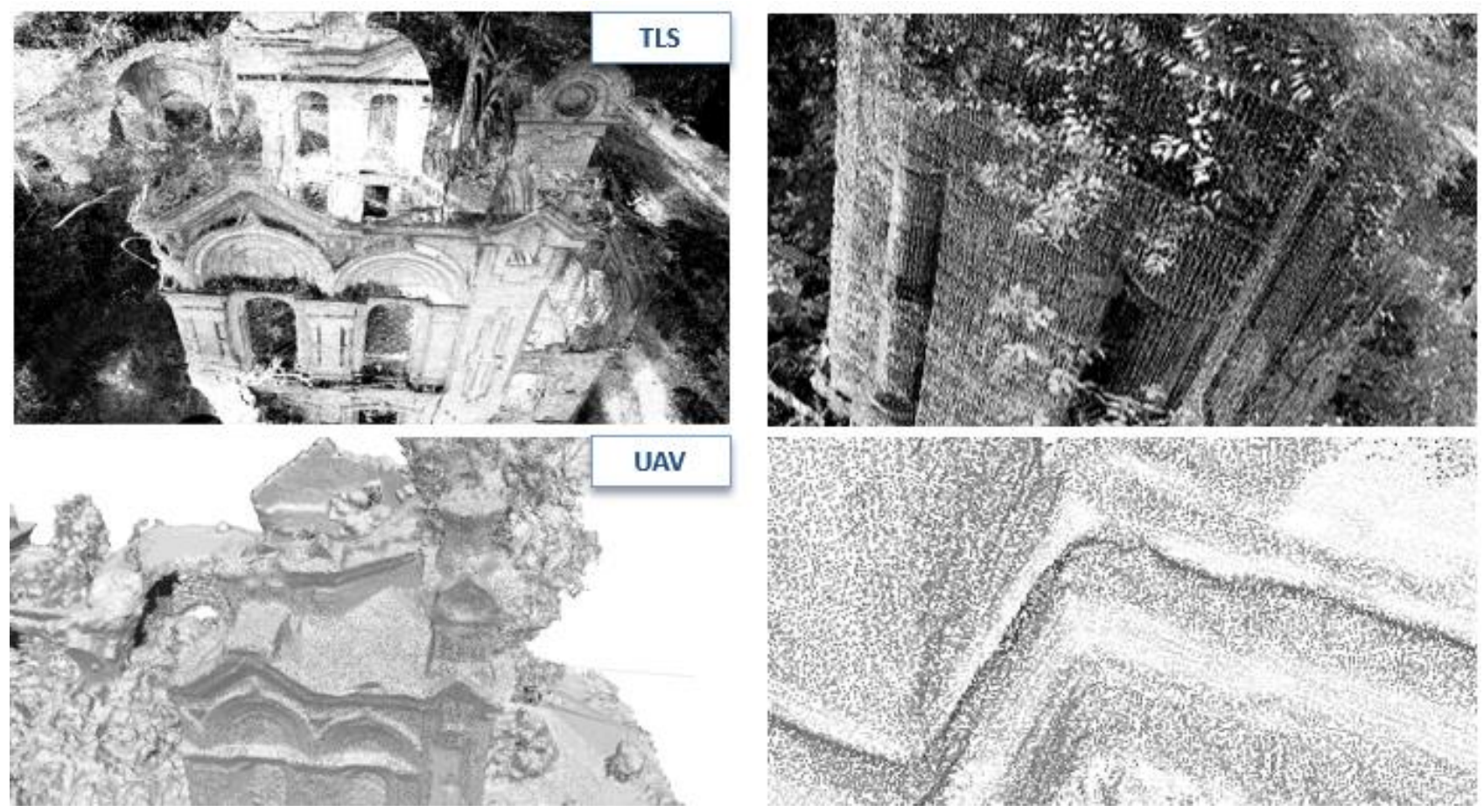

Figure 8. Quality of processed morpho-metric TLS and UAV data on the external surfaces of the masonry structure. The point cloud from the UAV acquisition shows a resolution and density of data comparable with the TLS acquisition, supported by the higher level and resolution of the camera shooting.

choice was oriented both toward a spatial configuration of the complex's elements, articulated on several levels and environments, and for a correspondence of the actual state of ruin of the building, which highlighted various critical issues to be transposed automatically in terms of a digitised model through non-uniform rational basis spline (NURBS) or parametric modelling. First, the data collected from the TLS and UAV acquisition campaigns were tested using a compatible procedure for the integration of the processed point clouds, also supported by common format extensions for their inclusion in the modelling platforms. During this phase, various issues emerged in the specific management of the vertex and polygon objects.

Corrections and uniformities of the normal vertex data

The first aspect related to the difficulty of transferring the 'vertex normal' information for each point of the photogrammetric cloud acquired via the UAV on the mesh modelling platforms. The compatible formats (mainly .ptx and .pts) for the reverse modelling provided a different ASCII code
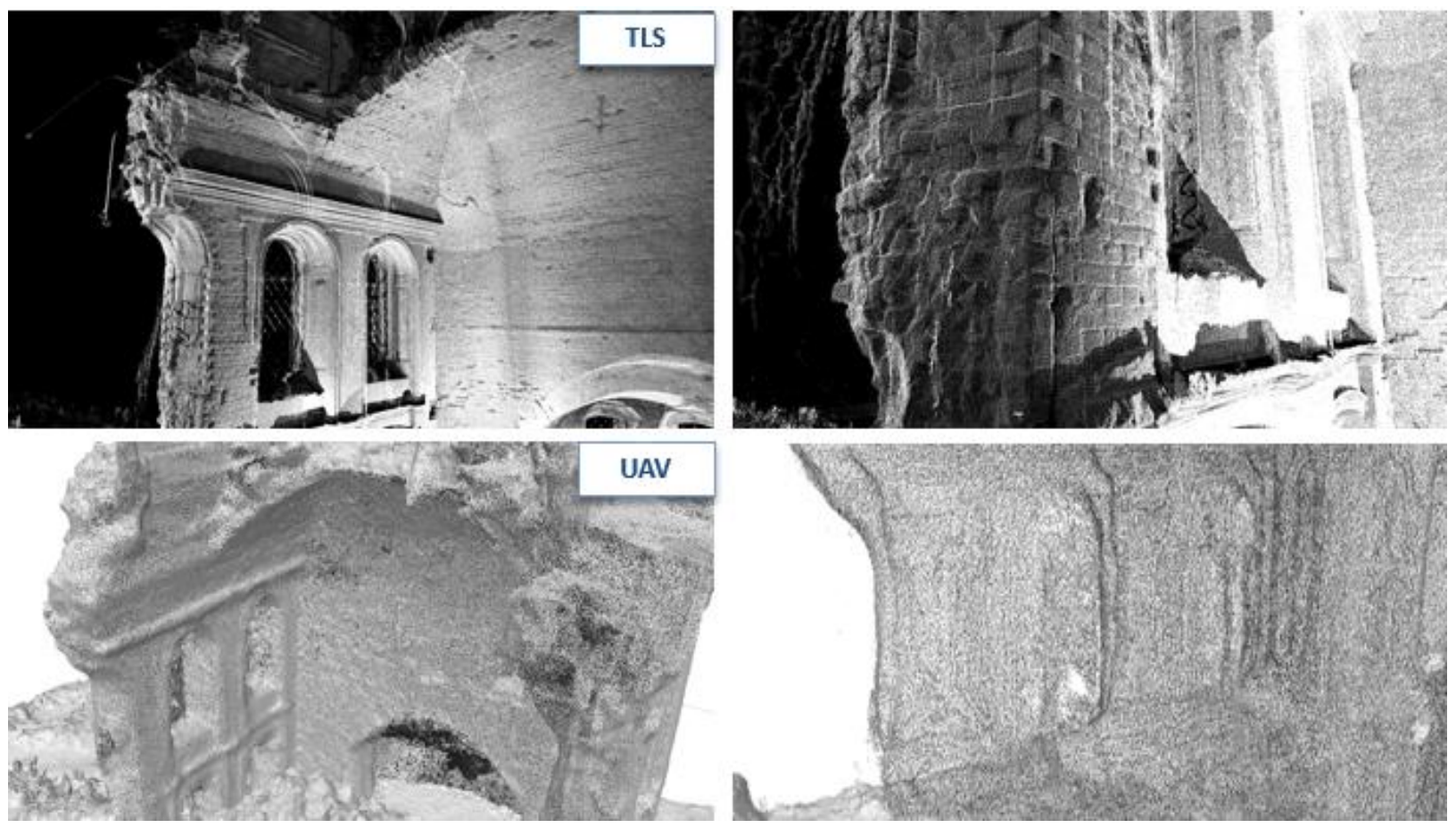

Figure 9. Quality of processed morpho-metric TLS and UAV data on the internal surfaces of the masonry structure. The point cloud from the UAV acquisition shows a worse resolution in the discrete definition of the main profiles, geometries, and surfaces, largely due to the corner and shadow shooting. 

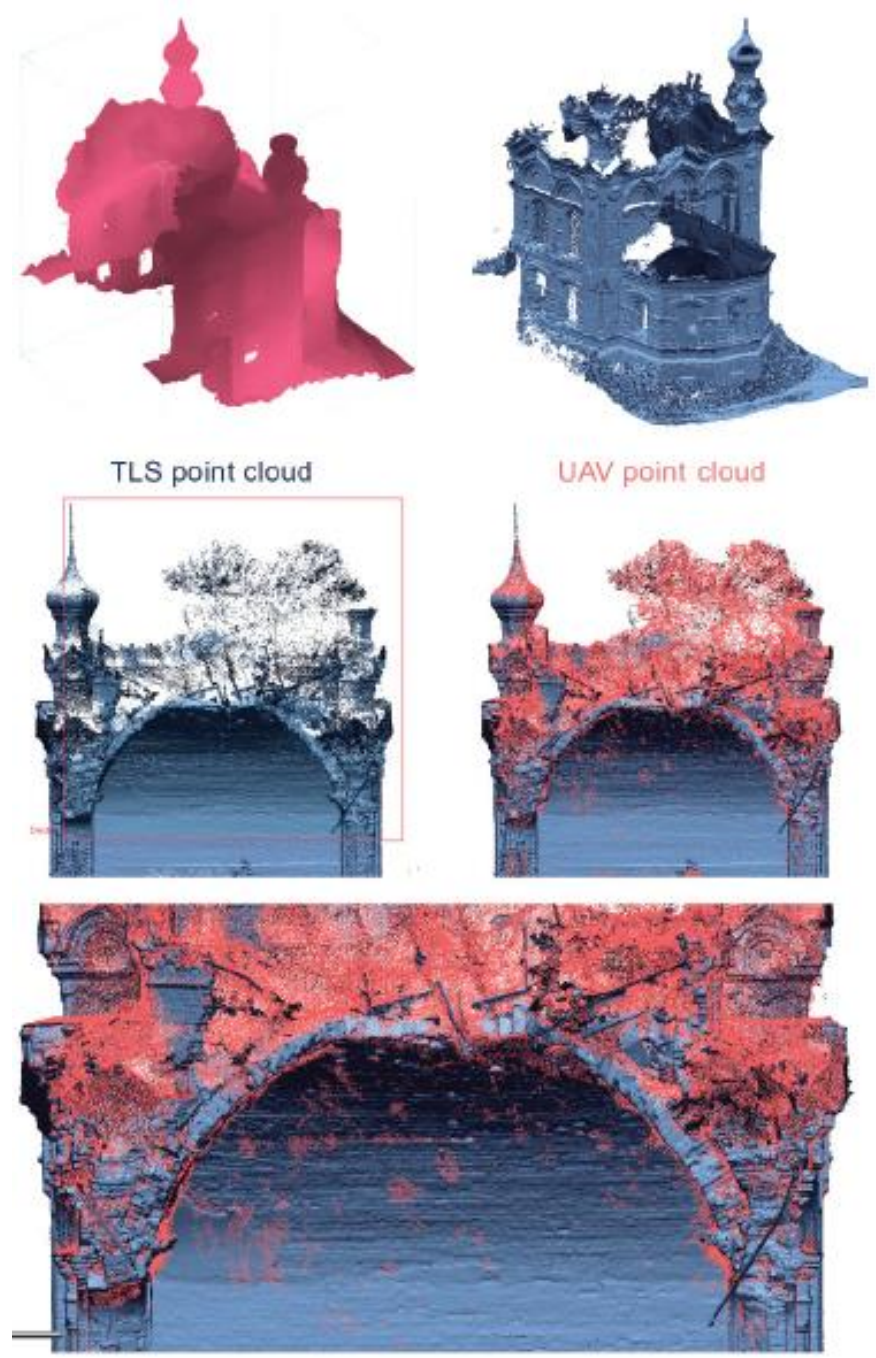

Figure 10. Alignment and integration of multi-instrumental point clouds for the static unit of the central pavilion vault.

in the UAV photogrammetric processing environment, and the normal information was corrupted within the different matrixes of conversion. Following various attempts at comparisons on the multiple modelling platforms suitable for the managing of architectural scale data, an additional phase of the re-calculation of the normal vertex in the UAV point cloud was adopted and performed directly in the modelling environment, so as to correctly orient the mesh triangulation and to avoid anomalies of flipped polygon regions that are not suitable for the mesh management as a whole. Due to the high density of points and their unstructured distribution, further complicated by the geometric articulation of the complex, the automatic normal calculation tools did not return uniform and coherent results, fragmenting the orientation of the points and, consequently, the features of the triangulated mesh. As such, only $70 \%$ of the points in the UAV-based point cloud (1.426.256 points) were automatically processed for the normal feature.

To resolve this issue, a single-step triangulation process was performed with the TLS and UAV integrated point cloud such that the normal vertex data defined in the TLS point cloud could mediate the orientation of the entire mesh surface. The topological meshing procedure was performed using an HD adaptive triangulation set for the following parameters:
Geometry capture accuracy: set over $75 \%$ of the details for shape accuracy.

Scanner accuracy: this related to the overall accuracy guaranteed by the source point cloud. Given the source TLS and UAV data, and the quality/noise of the integrated aligned database, the accuracy was set to within $3 \mathrm{~mm}$.

Filtering and selection of source data for the integration procedure.

The second aspect related to the different point spacing interval of the individual point clouds (on average, $1-3 \mathrm{~mm}$ from the TLS, and 5-10 $\mathrm{mm}$ from the UAV) and its influence on the triangulation of an overall polygonal mesh, where a uniform reading of structural irregularities was ensured. The two discontinuous datasets were refined with an overall filtering performed on the point clouds and were checked via a 'watertight remesh' process on the final mesh, assuming an average edge length of $5 \mathrm{~mm}$ to define a final model compatible with the main standards of deformation analysis in the area of structural diagnosis. This range did not compromise the relative dimensions of the structural damage quantifiable as a whole and related to the geometry of the construction materials (mainly bricks and metal chains of larger specific dimensions) and to the tolerance threshold set for the analysis $(5 \mathrm{~mm}$, also compatible with the standards for elastic safety assessments), Figure 11.

The finalisation of the integrated 3D survey database related to the Pokcha complex defined a virtual system of the preserved shape, directing the attention toward the metric-spatial correspondence of information obtained from the TLS database and the UAV photogrammetry, calibrated at different reliability values of space reconstruction from the features of the instruments applied in the acquisition phase. Specifically, a morphological reference and registration was developed in terms of the scale of each structural unit of the built complex. Here, for the pavilion vault, the two types of data were aligned in terms of perimetrical boundaries and façades while considering the deviation accuracy of the discreate surfaces and target control points. Following this, a segmentation of the overlapped point cloud was performed, deleting the overlapped areas and maintaining the quality of the TLS data on the intrados surfaces and both the TLS and UAV data on the extrados surfaces.

Finally, the set of points was subjected to standard filtering procedures, both automatic and manual, which were aimed at cleaning all the spatial information not pertaining to the envelope of the structural surface, specifically constituted by the infiltrated vegetation both at the base and at the top of the ruins. Further attention was paid to the window openings, which were manually cleaned for a complete reconstruction of the wall thickness. The triangulation phase of the final integrated database highlighted certain pieces of missing morphological information, which was due to the building masonry areas being covered by vegetation during the survey campaign (removed in the point cloud during the filtering process). These parts were subsequently integrated via a fitting of mesh holes according to the geometric primitives derived from the mesh model. The mesh model obtained by triangulating and remeshing the surfaces verified the expected target of morphological detail, conserving both the main structural profiles and the specific geometry qualities of the masonry elements, both on the surface and on the edges along the collapsed region. 


\subsection{Non-uniform rational basis spline comparison and deviation analysis}

The investigation into the structural instabilities was focused on the vaulted unit in the central span, with the aim of comparing the specific imperfect geometry of the vault (affected by mechanical deformations) with a reliable reconstruction of its original shape from the in-site dimensions of the ruins. Using the final mesh model, various section planes were set with an established pitch of $30 \mathrm{~cm}$ along all the surfaces of the unit (vault and vertical walls), extracting $3 \mathrm{D}$ sketch profiles that adhered to the specific morphological surface and importing them into a NURBS modelling environment. Using extrusion techniques, the structural volumes of the pavilion vault were geometrically reconstructed according to the regular project profiles and imported into the reverse modelling environment to allow for a comparison with the irregular mesh surface, Figure 12.

The comparison between the NURBS model and the mesh model of the vault was performed using the query tool of deviation available on the platform to compute the discrepancy between the models' surfaces. The deviation comparison (made possible by the same coordinate system maintained between the models) indicated an overall failure phenomenon that characterised the entire system of the resistant unit, possibly attributable to the accidental impact that caused its collapse. Within it, various specific portions affected by instability could be observed since their colour map obtained via the deviation computing was characterised by a colour scale ranging from green to red, highlighting the attendant on-course kinematic deformation that is increasing over time. These areas are located both on the vault and on the supporting walls, within deformations that are on a scale of centimetres and that are affecting the main loaded parts of the vaulted system, Figure 13.

Specifically, much like with the collapsed part, the remaining structure of the vault exhibits a discharging arch not in line with the overall static configuration. In fact, this shape reached $40 \mathrm{~mm}$ of deformation along entire height, it is subdivided into three plastic hinges and presents a serious risk of collapse. The main bearing walls also exhibit a buckling deformation that reached a maximum of $70 \mathrm{~mm}$, thus increasing the instability of the structure in the basement area.

As such, the reliable mapping of evolving structural instabilities using direct survey and mesh models is aimed at determining the intervention practices involving targeted consolidation actions for the safety and conservation of ruined structures.

\section{CONCLUSIONS}

The modelling performed in terms of the Church of the Annunciation in Pokcha followed an integrated approach involving morphometric data from different instrumental point clouds. An overall mesh triangulation strategy was devised to produce a reality-based model capable of preserving the structural irregularity through the mediation of numerical polygonal surfaces. Specific methodological considerations were developed for the mesh triangulation of the integrated TLS and UAV sparse databases. In order to perform an HD mesh construction, a correct alignment of the points was required as well as UAV point cloud processing to support the optimisation of the poly-face orientation in the mesh. Other filtering processes were implemented in terms of the point cloud, specifically regarding the presence of opening grids and extensive vegetation,
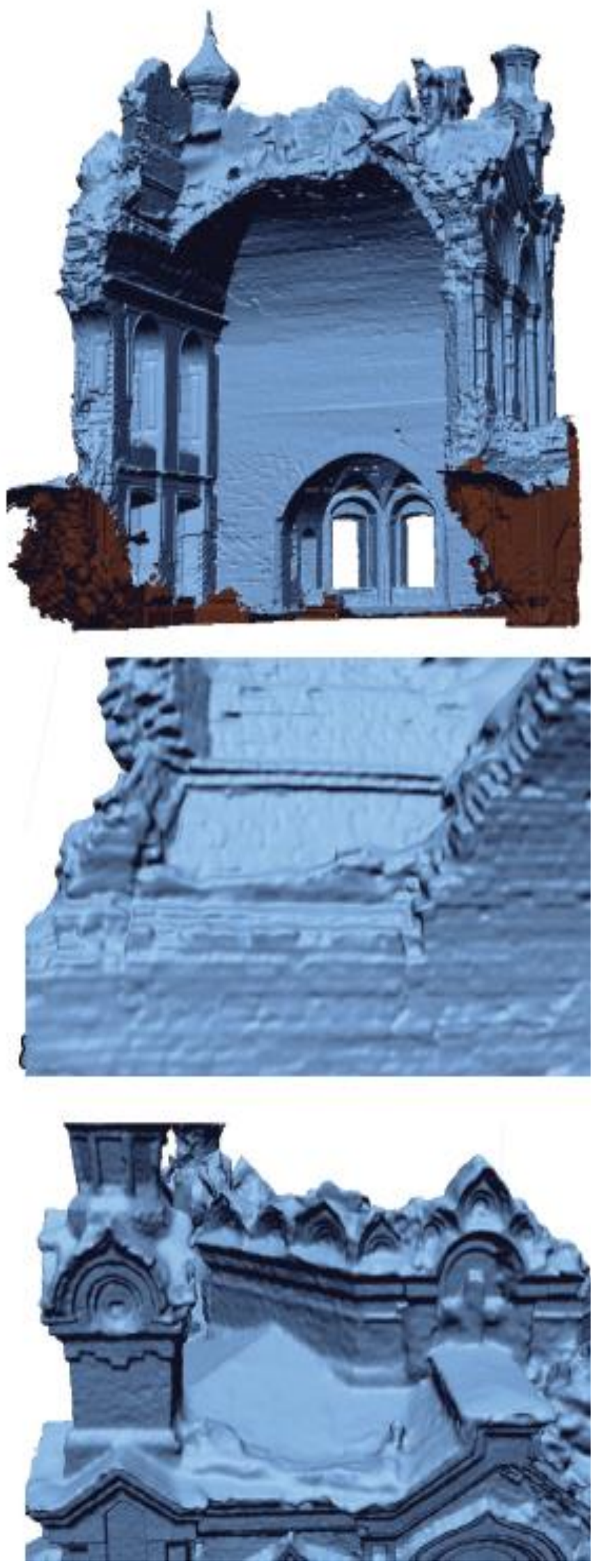

Figure 11. Final reality-based mesh models following the triangulation and optimisation processing of the HD mesh. The mesh preserved the specific details of the structural shape of the structural unit. 


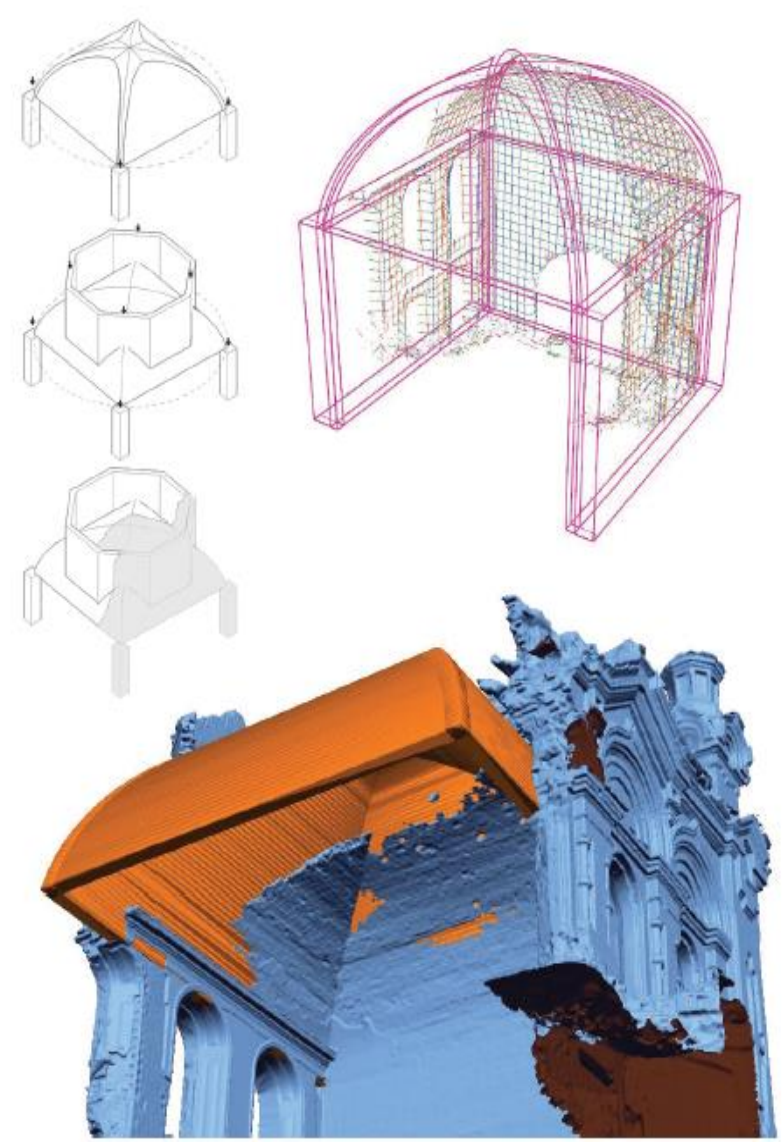

Figure 12. NURBS modelling of the pavilion vault from the sections extracted by the mesh surface. The geometric vault was referenced to the mesh model and was imported in the same workspace. to better expose the surface of the structural domain affected by the decay and the natural elements of the ruin site.

The need for a formal approach to the re-drawing analysis and intervention for endangered historical sites was the motivation behind the operational experimentation of morphologicalstructural representation in terms of two specific research targets:

1) The planning of a documentary strategy aimed at acquiring the totality and particularity of the architectural details in terms of all typological variants (masonry, metal parts, wall coverings) and collocations (main environments, underground, in elevation, coverage levels).

2) The convenience of transferring these detailed systems into suitable morpho-metric products capable of providing information and analytical opportunities in relation to historical masonries through graphic representations.

These objectives determined the preference for a 3D approach to the documentation and visualisation of structures directly from the data obtained via digital surveys. The shape representation is preserved in both qualitative and quantitative structural assessments, aware of the interactions that historical architecture can exhibit between its individual preserved components and, in terms of restoration, with the intervention design, Figure 14.

Due to its complex nature in terms of compatibility and format size, the type of morphometric data represented by highpoly mesh models is currently affected by the continuous developments in the field of Big Data management in relation to 3D databases pertaining to architectural systems and urban aggregates. This possibility for reverse modelling in terms of historical architectural heritage allows for revising the documentation protocols in a field of application of representative systems for structural diagnostics, to aspire to technical-professional adoption in the interface with mesh-to-
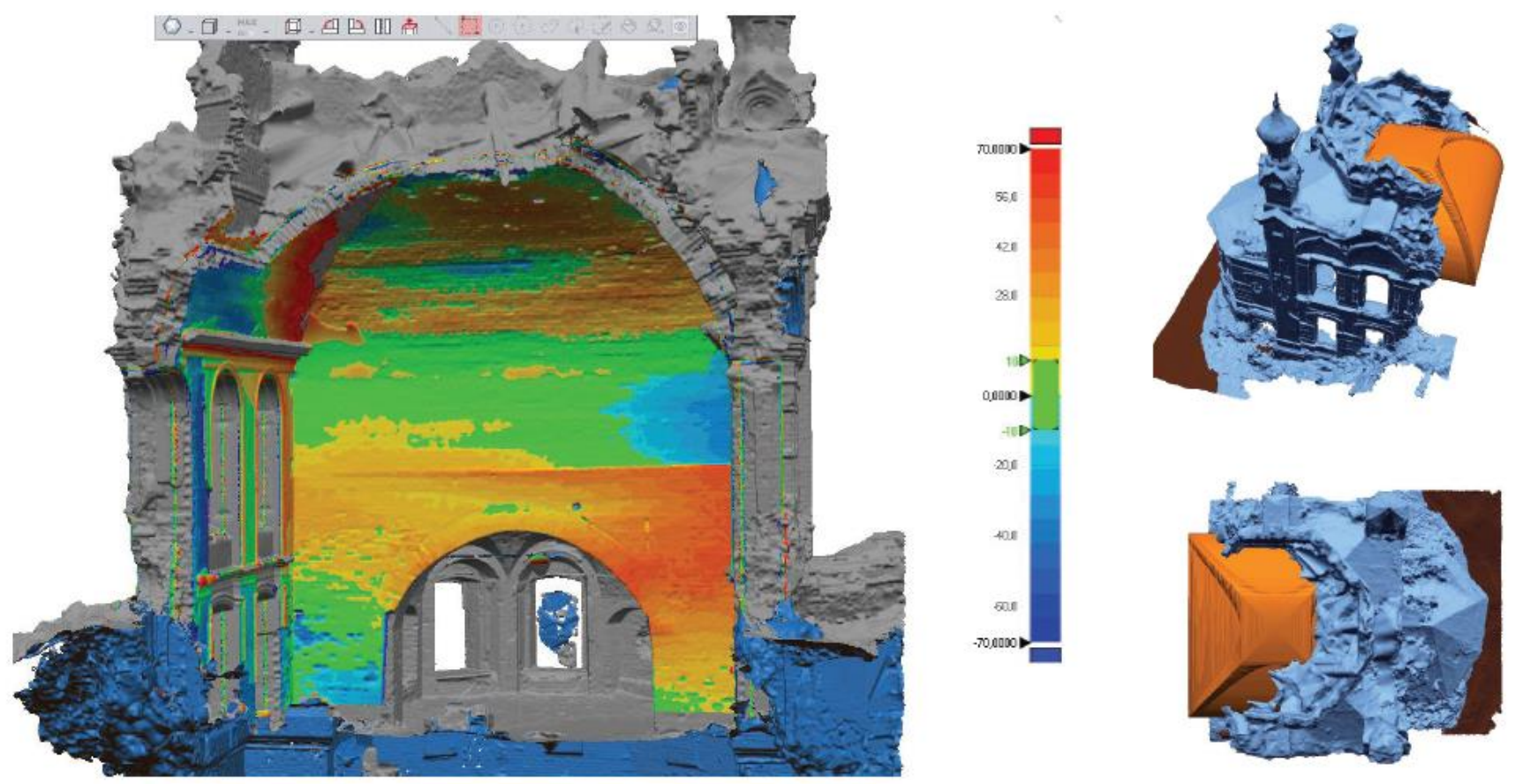

Figure 13. Deviation computing on the shape features between the models of the pavilion vault, from the HD mesh and from the NURB modelling. The colour map defines a quantitative reading of the deformed portions from the static configuration, highlighting the kinematic mechanisms that are affecting the vault. From the comparison, it was possible to identify the areas of instability and to derive diagnostic considerations in relation to the overall static schemes of the architectural complex as a whole. 

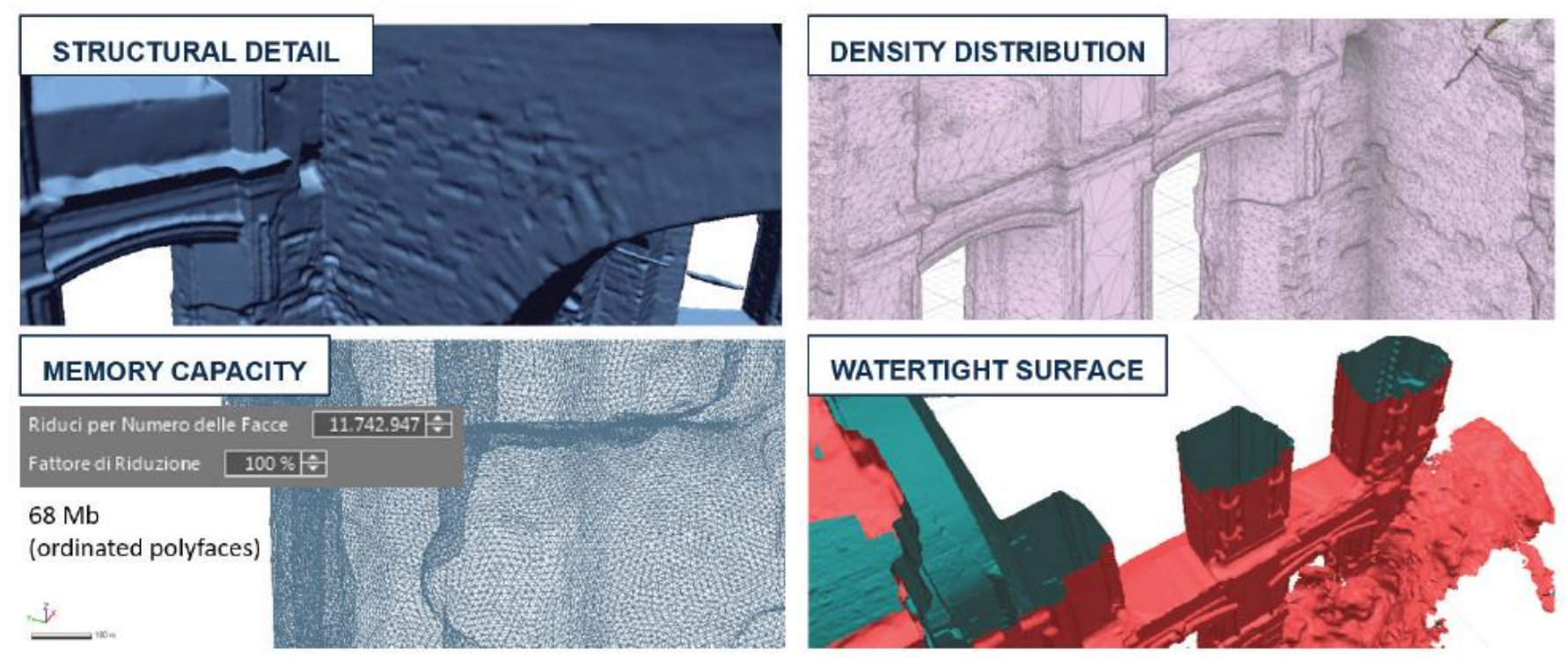

Figure 14. Properties of the final HD mesh model, which is suitable for compatible computing analysis in FEA workflows.

FEA and mesh-to-BIM protocols. Despite the national risk, the lines applied in the digital documentation demonstrate an attempt to adapt to the provisions of the Digital Agenda 2020 of the European Commission (also included in the Agenda Culture 2030), regarding the digitation of cultural heritage initiatives for the planning of intervention, restoration, and conservation. With the possibility of experimenting virtually within a simulated but reliable space, the methods and effects of a subsequent physical implementation of the intervention for heritage buildings emphasises the opportunities for digital preservation and eGovernance, expanding the ideas of innovation in relation to the type and quality of structural survey products in terms of the curation of digital assets and advanced digitisation. The action of 'structural' documentation, including the knowledge of stress behaviours and the prediction of damage mechanisms in buildings, can thus be identified within the European guidelines for heritage at risk by insisting on the characteristics of robustness.

\section{ACKNOWLEDGEMENTS}

The editorial authorship is granted to Sandro Parrinello for sections 1, 3 and the conclusions, and to Raffaella De Marco for sections 2,4 and 5 .

The documentation of the Upper Kama Region is part of a wider program of activities carried out, since 2013, by DAdaLAB - University of Pavia (coordinator prof. S. Parrinello) and the Perm National Research Polytechnic University (coordinator prof. S. Maximova). The digital survey campaigns of 2018 and 2019 were conducted by Parrinello S., Picchio F., De Marco R., Dell'Amico A. Part of the architectural survey documentation data was processed as part of the activities related to the course entitled 'Architectural survey \& restoration' (prof. S. Parrinello, prof. G. Minutoli) in the Double Degree Italian/Chinese course in Building Engineering and Architecture at the University of Pavia.

The surveying, documentation and modelling of Blagoveshchenskaya Church are part of the EU project, PROMETHEUS. This project is funded by the EU program Horizon 2020-R\&I-RISE-Research \& Innovation Staff Exchange Marie Skłodowska-Curie and involves the collaboration between three universities (University of Pavia,
Italy, Polytechnic University of Valencia, Spain, Perm National Research Polytechnic University Perm National Polytechnic University Research, Russia) and two companies (EBIME, Spain, SISMA, Italy).

This project has received funding from the European Union's Horizon 2020 research and innovation programme under Marie Skłodowska-Curie grant agreement No 821870.

\section{REFERENCES}

[1] R. de Koster (eds), Guidelines on Cultural Heritage. Technical tools for heritage conservation and management, JP - EU, 2012, Online [Accessed 26 March 2021]

https://rm.coe.int/16806ae4a9

[2] United Nations Educational, Scientific and Cultural Organisation, Operational Guidelines for the Implementation of the World Heritage Convention, WHC.17/01, UNESCO World Heritage Centre, Paris, France, 2017.

[3] UNESCO, Recueil de décisions importantes sur la conservation des biens du patrimoine culturel inscrits sur la Liste du patrimoine mondial en péril de IUNESCO, WHC-09/33.COM/9, Paris, 2009.

[4] K. Rao, A new paradigm for the identification, nomination and inscription of properties on the World Heritage List, International Journal of Heritage Studies 16 (2010), pp. 161-172.

DOI: $10.1080 / 13527251003620594$

[5] L. Toniolo, M. Boriani, G. Guidi (eds), Built Heritage: Monitoring Conservation Management, Springer, Cham., Switzerland, 2015, ISBN 978-3-319-08533-3. DOI: 10.1007/978-3-319-08533-3

[6] E. Psychogiopoulou, Cultural Heritage and the EU: Legal Competences, Instrumental Policies, and the Search for a European Dimension, in: A. Jakubowski, K. Hausler, F. Fiorentini (editors), Cultural Heritage in the European Union. A Critical Inquiry into Law and Policy, Brill Nijhoff, Netherlands, 2019, ISBN: 978-90-04-36534-6, pp. 57-78.

DOI: $\underline{10.1163 / 9789004365346012}$

[7] S. Parrinello, R. De Marco, From the city to the stone: digital survey for the establishment of structural behaviours in historical architecture, in: R. Salerno, Drawing as (in)Tangible Representation, Gangemi Editore, Roma, Italy, 2018, ISBN 97888-492-3651-4, pp. 747-754.

[8] B. M. Feilden, J. Jokilehto, Management Guidelines for World Cultural Heritage Sites, ICCROM, Rome, Italy, 1998. ISBN: 929077-150-X

[9] M. Bruneau, S. E. Chang, R. T. Eguchi, G. C. Lee, T. D. ORourke, A. M. Reinhorn, M. Shinozuka, K. Tierney, W. A. Wallace, D. von 
Winterfeldt, A framework to quantitatively assess and enhance the seismic resilience of communities, Earthquake Spectra 19(4) (2003), pp. 733-752.

DOI: http://dx.doi.org/10.1193/1.1623497

[10] A. Giuffrè, C.F. Carocci, Statics and dynamics of historical masonry buildings, in: proceedings International Workshop on Structural Restoration of Historical Buildings in Old City Centers, Heraklion, Crete, 1994, pp. 71-152.

[11] A. M. DAltri, G. Milani, S. de Miranda, G. Castellazzi, V. Sarhosis, Stability analysis of leaning historic masonry structures, Automation in Construction Volume 92 (2018), pp. 199-213. DOI: $10.1016 /$ i.autcon.2018.04.003

[12] F. Parisi, N. Augenti, Earthquake damages to cultural heritage constructions and simplified assessment of artworks, Engineering Failure Analysis 34 (2013), pp. 735-760. DOI: $10.1016 /$ i.engfailanal.2013.01.005

[13] S. Parrinello, F. Picchio, P. Becherini, R. De Marco, The drawn landscape in $3 \mathrm{~d}$ databases: the management of complexity and representation in the historical city, 7th Annual International Conference on urban Studies \& Planning, Atiners Conference Paper Series, Athens, 2018, pp. 3-26.

DOI: $10.30958 /$ aja.4-3-2

[14] S. Parrinello, F. Picchio, R. De Marco, A. DellAmico, Documenting the Cultural Heritage Routes. The creation of informative models of historical Russian churches in the Upper Kama Region. The International Archives of the Photogrammetry, Remote Sensing and Spatial Information Sciences XLII-2/W15 (2019), pp. 887-894. DOI: 10.5194/isprs-archives-XLII-2-W15-887-2019

[15] W. C. Brumfield, Landmarks of Russian Architecture, Gordon e Breach, Amsterdam, 1997.

[16] E. N. Shumilov, Orthodox Churches and Temples of Common Faith in Perm Krai: short historical guide, PKUB, Perm, 2003.

[17] L. A. Shatrov, Monuments of architecture, history, art of Cherdyn and Cherdyn Region, V.2. Cherdyn, 1997.

[18] A. Guarnieri, F. Pirotti, M. Pontin, A. Vettore, Combined 3D surveying techniques for structural analysis applications, Proc. of International Symposium on Photogrammetry and Remote Sensing (ISPRS), 2005, vol. XXXVI-5/W1, pp. 22-24.

[19] S. Parrinello, F. Picchio, R. De Marco, New generations of digital databases for the development of architectural urban risk management, in: C. Gambardella, Le Vie dei Mercanti XV Forum Internazionale World Heritage and Disaster, Fabbrica della Conoscenza, vol. 71, 2017, ISBN: 978-88-6542-582-4, pp. 1-10.
Online [Accessed 26 March 2021]

http://www.leviedeimercanti.it/proceedings-xv-forum

[20] M. Tsakiri, D. Lichti, N. Pfeifer, Terrestrial laser scanning for deformation monitoring, in: Proceedings of 3rd IAG/12th FIG Symp., Institute of Geodesy and Geophysics, Baden, Germany, 2006, pp.1-10. Online [Accessed 26 March 2021] https://www.fig.net/resources/proceedings/2006/baden 2006 comm6/PDF/LS2/Tsakiri.pdf

[21] A. Guarnieri, N. Milan, A. Vettore, Monitoring of complex structure for structural control using terrestrial laser scanning (TLS) and photogrammetry, International Journal of Architectural Heritage 7(1) (2013), pp. 54-67. DOI: $\underline{10.1080 / 15583058.2011 .606595}$

[22] S. Bertocci, G. Minutoli, G. Pancani, 3D survey and instability analysis of Romena parish. Disegnare CON 8(14) (2015), pp. 1-20 Online [Accessed 26 March 2021] http://disegnarecon.univaq.it/ojs/index.php/disegnarecon/artic le/view/36/31

[23] R. De Marco, Shapes and models: the survey for the study of structures in historical buildings, in: K. Williams, M. G. Bevilacqua. Nexus 2018 Architecture and Mathematics Conference Book. Kim Williams Books, Pisa, Italy, 2018, ISBN 978-88-88479-47-7, pp. 289-292.

[24] L. De Luca, P. Veron, M. Florenzano, Reverse-engineering of architectural buildings based on an hybrid modeling approach, Computers \& Graphics 30(2) (2006), pp. 160-176. Online [Accessed 26 March 2021] https://hal.archives-ouvertes.fr/hal-01021897

[25] G. Castellazzi, A. M. DAltri, S. de Miranda, F. Ubertini, An innovative numerical modeling strategy for the structural analysis of historical monumental buildings, Engineering Structures 132 (2017), pp. 229-248

DOI: $10.1016 /$ i.engstruct.2016.11.032

[26] M. Centofanti, S. Brusaporci, Architectural 3D modeling in historical buildings knowledge and restoration processes, in: $\mathrm{C}$. Gambardella, Le Vie dei Mercanti, X Forum Internazionale di Studi, More or Less. Fabbrica della Conoscenza, vol. 16, 2012, ISBN 978-88-6542-129-1, pp. 331-340. Online [Accessed 26 March 2021] http://www.leviedeimercanti.it/proceeding-x-forum/

[27] S. Parrinello, R. De Marco. From survey to the model: the graphic transposition of an earthquake, Disegnare Idee Immagini 57 (2018), pp. 70-81. 